\title{
Study of Optical Properties of (PMMA-CuO) Nanocomposites
}

\author{
Bahaa H. Rabee, Bairaq Abd Al-Kareem \\ Babylon University, College of Education for Pure Science, Department of Physics, Iraq
}

\begin{abstract}
The effect of addition the copper oxide nanoparticles on optical properties of polymethylmethacrylate has been studied. For this purpose, many samples have been prepared by adding copper oxide nanoparticles to with different weight percentages by using casting method. The absorption spectra has been recorded at the wavelength ranges (300-1000) nm. The results show that the (absorption coefficient, extinction coefficient, refractive index and real and imaginary dielectric constants) of (PMMA-CuO) nanocomposites are increasing with the increase of the copper oxide nanoparticles concentrations. The energy band gap of (PMMA$\mathrm{CuO}$ ) nanocomposites decreases with the increase of the copper oxide nanoparticles concentrations.
\end{abstract}

Keywords: Nanocomposites, Copper Oxide Nanoparticles , Optical Properties

\section{Introduction}

Polymer nanocomposites have unique properties such as light weight, high flexibility, and ability to be fabricated at low temperature and low cost [1]. Polymers are of profound interest to society and are replacing metals in diverse fields of life, which can be further modified according to modern application. They are more desirable than traditional materials in such areas as packaging, construction, and medical applications. Processing of polymeric materials basically depends on applied heat and pressure [2].

Many of modern technologies require materials with unusual combinations of properties that cannot be met by the conventional metal, alloys, ceramics, and polymeric materials. This is especially true for materials that are needed for aerospace, underwater, and transportation applications[3]. A composite material is basically a combination of two or more materials, each of which retains its own distinctive properties. usually the term composite is applied to materials that are created by mechanical bonding two or more different materials together. The resulting materials have characteristics that are not characteristics of the component in isolation[4]. Nanotechnology is likely to have a profound impact on our economy and society in the early twenty-first century, science and technology research in nanotechnology promises breakthroughs in such areas as materials and manufacturing, nanoelectronics, medicine and healthcare, energy, biotechnology, information technology, and nationa security. It is widely felt that nanotechnology will be the next industrial revolution[5]. The main reasons for the continue increase in the demand of the commodity plastics are as follows:

- Plastics are low density solids, which makes it possible to produce lightweight objects.

- Plastics have low thermal and electric conductivities, since they are widely used for insulation purposes.

- Plastics are easily moulded into desired shapes.

- Plastics usually exhibit high corrosion resistance and low degradation rates and are highly durable materials.

- Plastics are low cost materials.

All these advantages make the plastic materials to be used in almost all fields of the every day life[6]. Poly(methyl methacrylate) is an amorphous thermoplastic, PMMA is produced by the addition polymerization of methyl methacrylate. The polymer has very good optical properties but has poor scratch resistance. It has good dimensional stability due to rigid polymer chains. It has good weather resistance, and is stable to acid and alkalis. It is attacked by several organic solvents, and has good impact strength higher, than that of glass or polystyrene. It has the best transparency and optical properties of commercially available thermoplastic[7]. PMMA is a colorless transparent plastic, i.e. transmits light almost perfectly (92\%), which make them suitable to serve as a conduit for light[8]. Chloroform, which is used as a solvent for PMMA, is considered as the greatest soluble limit for PMMA; it has the greatest evaporation rates, less viscosity and the least chemical hazard than other solvents [9].

Copper oxide $(\mathrm{CuO})$ nanoparticles are prominent due to their varied applications in superconductors, optical, electrical, catalytic, photocatalytic degradation, gas sensors, and in biosensors. $\mathrm{CuO}$ is an antimicrobial, anti-biotic and antifungal agent when incorporated in coatings, plastics, textiles, etc. It acts as a catalyst to eliminate industrial effluent in the environment. The elimination of these pigments and other organic materials ensures a safe and clean environment [10]. The optical properties of amorphous semiconductors have been the subject of many recent papers. The study of the optical constants of materials composite is interesting for many reasons. First, the use of materials in reflected coating requires accurate knowledge and optical fibers of their optical constants over wide ranges of wavelength. Second, the optical properties of all materials are related to their electronic band structure, atomic structure and electrical properties. The optical properties of polymers can be suitably modified by the addition of dopants depending on their reactivity with the host matrix[11].

The aim of this paper, the effect $\mathrm{CuO}$ nanoparticles on the optical properties of the Polymethylmethacrylate.

\section{Experimental Part}

Polymethylmethacrylate solution was prepared by dissolving it in chloroform by using magnetic stirrer in mixing process 


\section{International Journal of Science and Research (IJSR) \\ ISSN (Online): 2319-7064 \\ Index Copernicus Value (2013): 6.14 | Impact Factor (2015): 6.391}

to get homogeneous. Nanocomposites of (PMMA-CuO) films are prepared by using casting method. The copper oxide nanoparticles are added to poly (methyl methacrylate) with different concentrations are (0,2,4,6 and 8) wt.\% and mixed for 20 minutes to get more homogenous solution. The optical properties of (PMMA- CuO) nanocomposites are measured by using UV/1800/ Shimadzu spectrophotometer in range of wavelength (320-1040) $\mathrm{nm}$. The optical constants are very important because they describe the optical behavior of the materials. The absorption coefficient of the material is very strong function of photon energy and band gap energy[12].

Absorptance (A) is defined as the ratio between absorbed light intensity $\left(\mathrm{I}_{\mathrm{A}}\right)$ by material and the incident intensity of light $\left(I_{0}\right)$ [13].

$$
\mathrm{A}=\mathrm{I}_{\mathrm{A}} / \mathrm{I}_{\mathrm{O}}
$$

Transmittance (T) is given by reference to the intensity of the rays transmitting from the film (I) to the intensity of the incident rays on it $\left(\mathrm{I}_{0}\right)(\mathrm{T}=\mathrm{I} / \mathrm{Io})$, and can be calculated by:

$$
\mathrm{T}=\exp (-2.303 \mathrm{~A})
$$

And Reflectance (R) can be obtained from absorption and transmission spectra in accordance with the law of conservation of energy by the relation:

$$
R+T+A=1
$$

\section{Absorption coefficient $(\alpha)$ of (PMMA-CuO)} nanocomposites is defined by following equation[18]:

$$
\alpha=2.303 \mathrm{~A} / \mathrm{t}
$$

Where A: is the absorbance and t: is the sample thickness.

The indirect transition for amorphous materials is[14]:

$$
\alpha h v=B\left(h v-E_{g}\right)^{r}
$$

Where B is a constant, hv is the photon energy, $E_{g}$ is the optical energy band gap, $\mathrm{r}=2$ for allowed indirect transition and $r=3$ for forbidden indirect transition.

The Refractive index (n) is given by following equation for (PMMA-CuO) nanocomposites[15]:

Where $\mathrm{R}$ is the reflectance.

$$
n=\left(1+R^{1 / 2}\right) /\left(1-R^{1 / 2}\right)
$$

The extinction coefficient $(\mathrm{k})$ is calculated by the following equation[15]:

$$
\mathrm{K}=\alpha \lambda / 4 \pi
$$

The dielectric constants $(\varepsilon)$ is divided into two parts real $\left(\varepsilon_{1}\right)$ and imaginary $\left(\varepsilon_{2}\right)$ are calculated by using equations [16]:

$$
\begin{gathered}
\varepsilon_{1}=\mathrm{n}^{2}-\mathrm{k}^{2} \\
\varepsilon_{2}=2 \mathrm{nk}
\end{gathered}
$$

\section{Results and Discussion}

The variation of absorbance of (PMMA-CuO) nanocomposites with wavelength of different concentrations of additive nanoparticles is shown in figure(1). The figure shows that the absorbance of polymer is increased with the increase the concentrations of nanoparticle, this is due to the nanoparticles absorb the incident light[17].

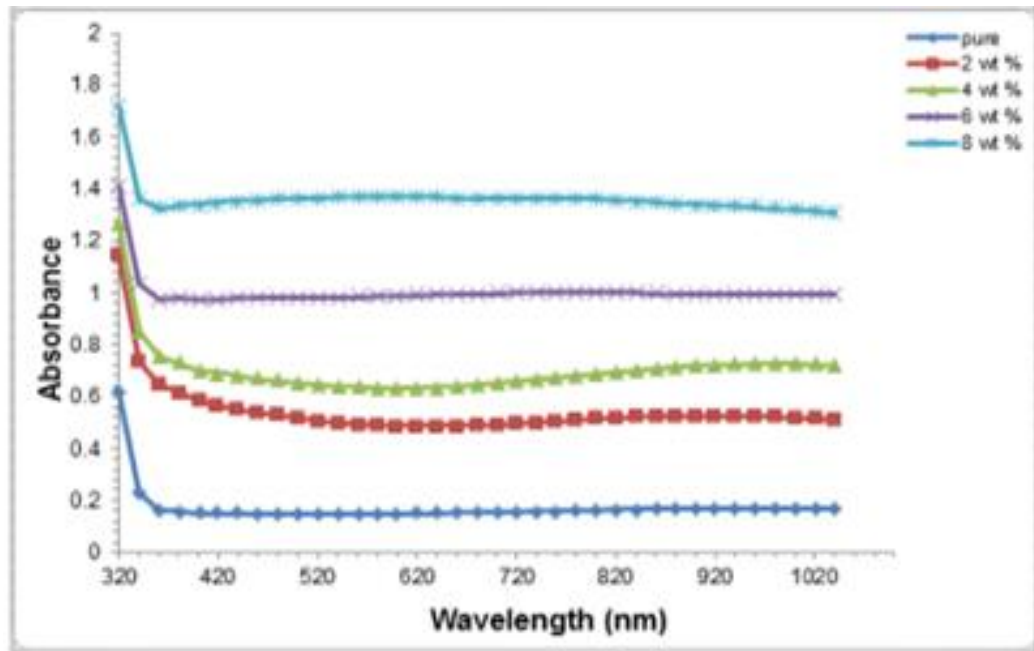

Figure 1: Variation of the absorbance of (PMMA-CuO) nanocomposites with the wavelength

Figure (2) shows the optical transmittance spectra as function of incident wavelength for (PMMA-CuO) nanocomposites. From the figure, the transmittance decreases with increasing of concentration of nanoparticles. 


\section{International Journal of Science and Research (IJSR) \\ ISSN (Online): 2319-7064}

Index Copernicus Value (2013): 6.14 | Impact Factor (2015): 6.391

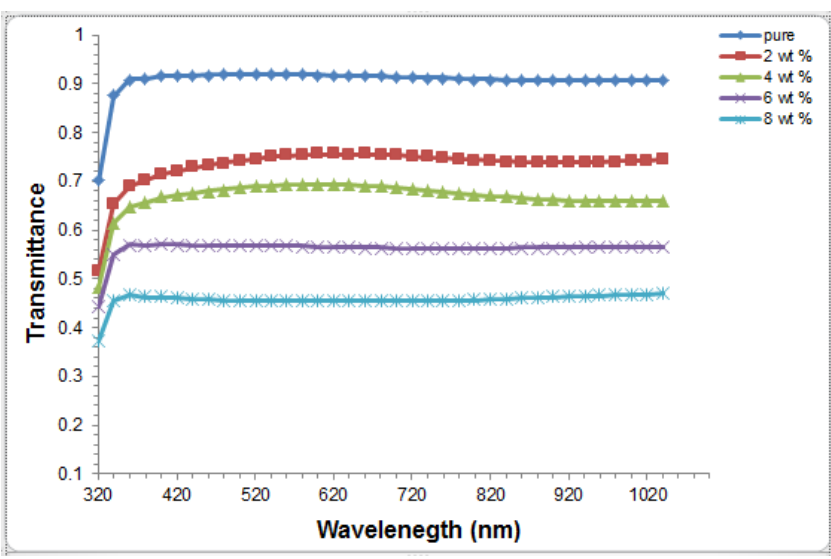

Figure 2: variation of the transmittance of (PMMA-CuO) nanocomposites with the wavelength

The relationship between the absorption coefficient of (PMMA-CuO) nanocomposites and photon energy with different weight percentages of copper oxide nanoparticles is shown in figure (3). From the figure, the absorption coefficient of (PMMA-CuO) nanocomposites increases with the increase of the copper oxide nanoparticles concentrations which related to the increase the number of carries charges in nanocomposites[18].

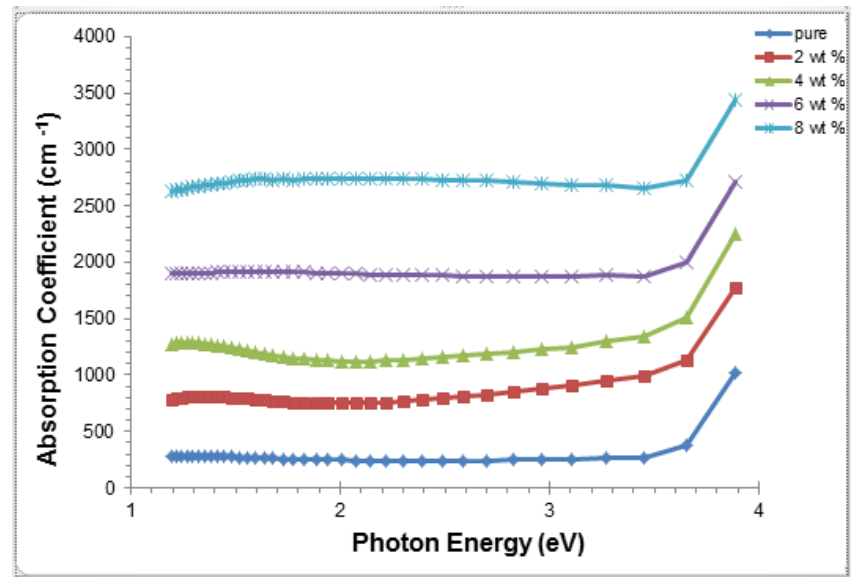

Figure 3: Variation of the absorption coefficient of (PMMA-CuO) nanocomposites with photon energy.

The values of absorption coefficient of (PMMA-CuO) nanocomposites are less than $10^{4} \mathrm{~cm}^{-1}$, so, the nanocomposites have indirect energy gap as shown in figure (4) and figure (5) for allowed indirect and forbidden indirect transition of (PMMA-CuO) nanocompsites respectively. The energy band gap of (PMMA-CuO) nanocompsites decreases with the increase of the concentrations for copper oxide nanoparticles, this behavior attributed to the increase of the localized level in energy band gap[19].

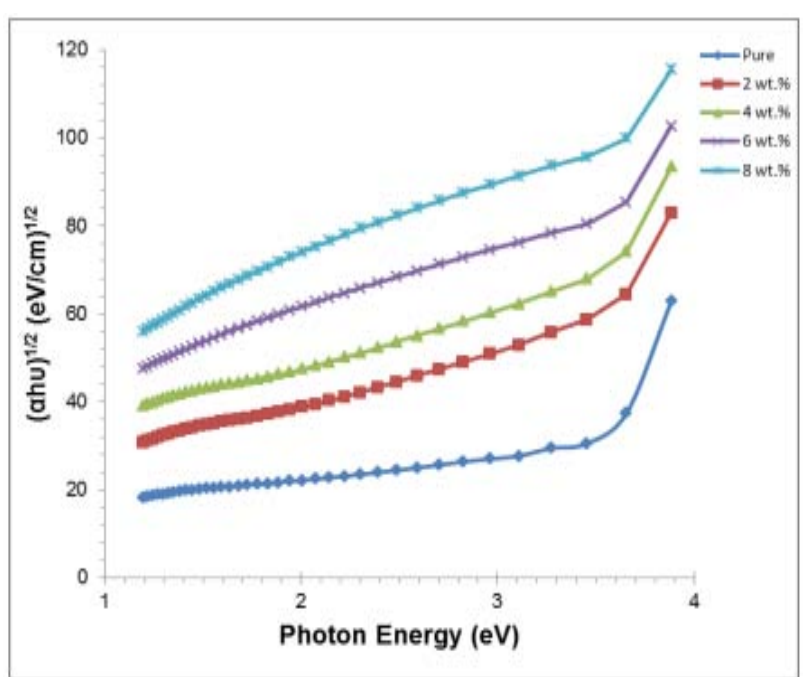

Figure 4: Relationship between $(\alpha h v)^{1 / 2}\left(\mathrm{~cm}^{-1} . \mathrm{eV}\right)^{1 / 2}$ and photon energy of (PMMA-CuO) nanocomposites.

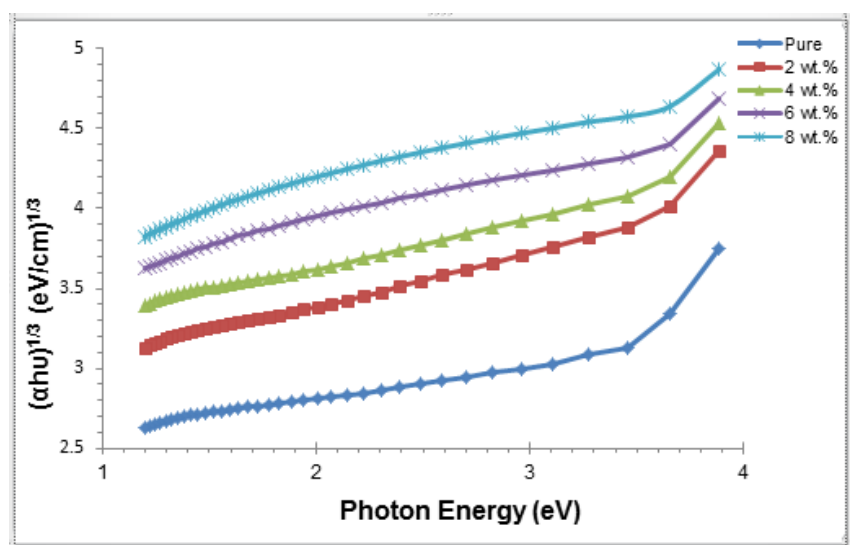

Figure 5: relationship between $(\alpha h v)^{1 / 3}\left(\mathrm{~cm}^{-1} . \mathrm{eV}\right)^{1 / 3}$ and photon energy of (PMMA-CuO) nanocomposites.

Figure (6) shows the variation of the extinction coefficient of nanocompsites with photon energy for different concentration of copper oxide nanoparticles. The extinction coefficient is increased with the increase of copper oxide nanoparticles concentrations which attributed to the increase of absorb part of the incident light[20], and so, loss of energy because the reaction between the light and the molecules of the medium[21].

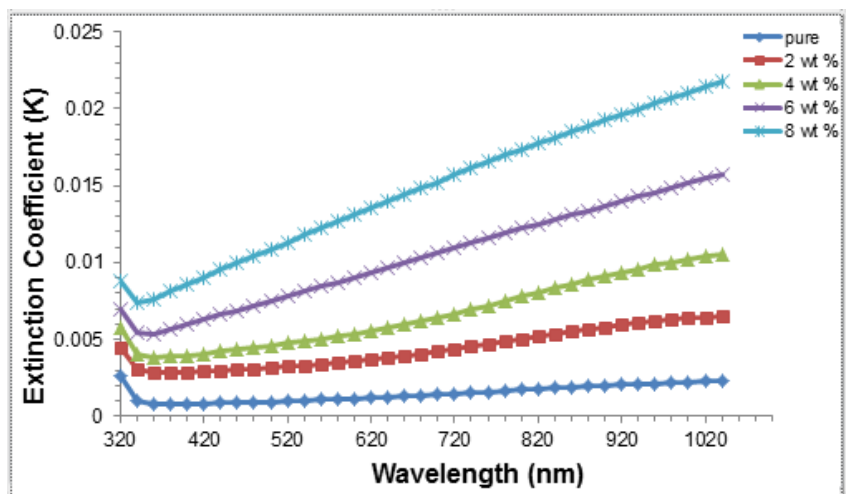

Figure 6: variation of extinction coefficient of (PMMA-CuO) nanocomposites with the wavelength

The relationship between the refractive index of (PMMA$\mathrm{CuO}$ ) nanocomposites and wavelength is shown in figure (7). 


\section{International Journal of Science and Research (IJSR) \\ ISSN (Online): 2319-7064 \\ Index Copernicus Value (2013): 6.14 | Impact Factor (2015): 6.391}

The figure shows that the refractive index increases with the increase of the concentration of filler which is due to the increase the scattering of the light [23]; so it increase, because the increase of the density of nanocomposite[22].

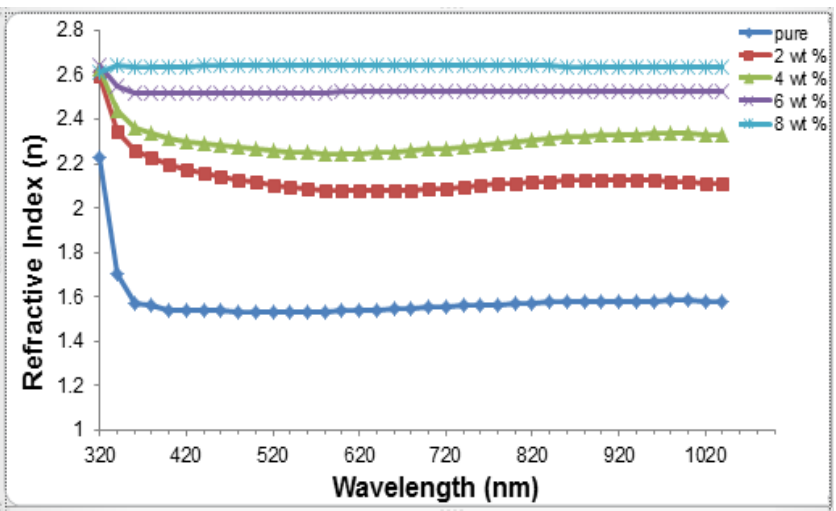

Figure 7: relationship between the refractive index of (PMMA-CuO) nanocomposites and wavelength.

Figure (8) and figure (9) show the variation of real and imaginary parts of dielectric constant of (PMMA-CuO) nanocompsites with wavelength for different $\mathrm{CuO}$ nanoparticles concentrations respectively. From the figures, the real and imaginary parts of dielectric constant for (PMMA-CuO) nanocomposites increase with the increase of $\mathrm{CuO}$ nanoparticles concentrations. The increase of real and imaginary parts of dielectric constant attributed to increase the absorption of incident light and the density of (PMMA$\mathrm{CuO}$ ) nanocomposites with the increase of copper oxide nanoparticles concentrations[23].

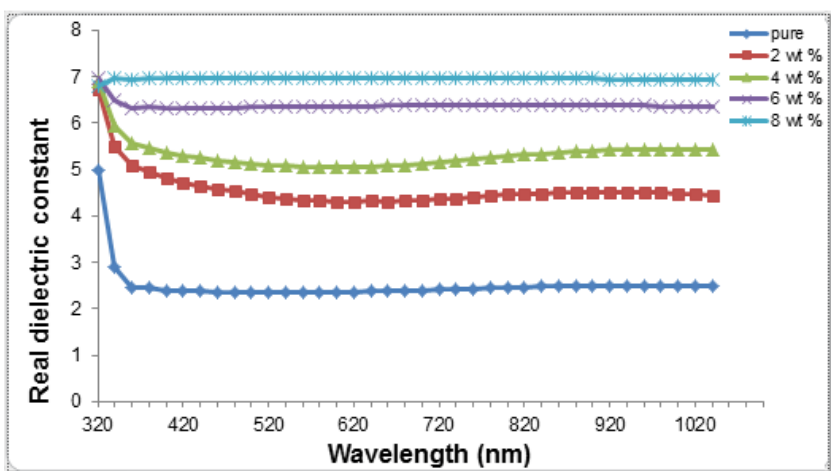

Figure 8: variation of real part of dielectric constant of (PMMA-CuO) nanocompsites with wavelength.

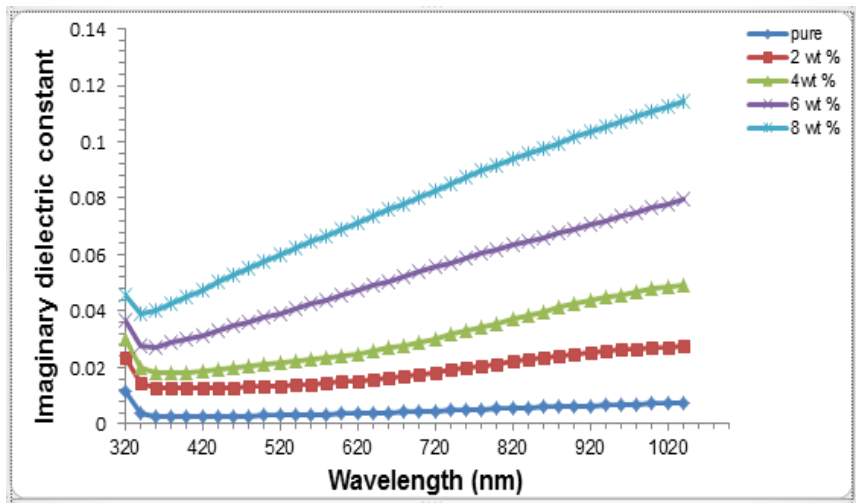

Figure 9: Variation of imaginary part of dielectric constant of (PMMA-CuO) nanocompsites with wavelength

\section{Conclusions}

1) The absorbance of (PMMA-CuO) nanocomposites increases with the increase of the concentrations of copper oxide nanoparticles and the transmittance decreases with increasing of the concentrations of the copper oxide nanoparticles.

2) The optical constants (absorption coefficient, extinction coefficient, refractive index and real and imaginary dielectric constants) of (PMMA-CuO) nanocomposites are increasing with the increase of the concentrations of the copper oxide nanoparticles.

3) The energy band gap of (PMMA-CuO) nanocomposites decreases with the increase of the concentrations of the copper oxide nanoparticles.

\section{References}

[1] P.U., Asogwa, 2011. "Band gap shift and optical characterization of PVA- Capped PbO thin films : Effect of thermal annealing " Chalcogenide Letters, 8(3): 163170.

[2] M. N. Subramanian, "Basics of Polymers Fabrication and Processing Technology" , India , Printed in the United States of America, Momentum Press ${ }^{\circledR}$, LLC, (2015).

[3] D.W. Callister , "Material Science and Engineering , An Introduction", Sixth Edition, Department of Metallurgical Eng., The University of Utah, John Wiley and Sons, Inc., USA, (2003).

[4] M. Arifitekhar, "Introduction to Composite Materials", BMEn 5001, (1999).

[5] Z.I., Ali, M.H. Helal, H.H. Saleh, A.F. Zikry, Y.A. Darwish, 2014. "Silver-Polymer Blend Nanocomposite", New York Science Journal, 7(1).

[6] IR Salagean, MC Pascariu, G Bandur, LM Rusnac. Thermal Properties of Copolymers Based on Sugar, Journal of Chem. Bull., 2009; 54(68): 100-103.

[7] J. H. Nahida , "Spectrophotometric Analysis for the UVIrradiated (PMMA)" , International Journal of Basic \& Applied Sciences , (12) 2 , pp. 127102-5454 , (2012).

[8] M. N. Al- Dulaimi,"The Effects of $\mathrm{Ni}, \mathrm{Cu}$, and CB Additives on the Electrical ,Optical and Mechanical Properties of PMMA" ,Ph. D. Thesis Physics Dep . Faculty of Science University of Baghdad, (1996).

[9] Y.F., Qian, Y. Su, X.G. Li, H.S. Wang and C.L.He,2010. Electrospinning of Polymethyl Methacrylate Nanofibres in Different Solvents, Iranian Polymer Journal,19(2): 123-129.

[10] K.L.Niraimathi , R.Lavanya , V.Sudha , R.Narendran and P.Brindha , "Bio-Reductive Synthesis and Characterization of Copper Oxide Nanoparticles (CuONPs) Using Alternanthera sessilis Linn. Leaf Extract", Journal of Pharmacy Research , (10)1 , PP. 2932, (2016) .

[11]A., Ameer1, Mustafa S. Abdallh, Ahmed A. Ahmed, Emad A. Yousif, 2013. Synthesis and Characterization of Polyvinyl Chloride Chemically Modified by Amines", Open Journal of Polymer Chemistry, 3: 11-15.

[12] Pelaiz-Barranco, A.; Marin-Franch, P.; 2005"Piezo-, pyro-, ferro-, and dielectric properties of ceramic/polymer composites obtained from two modifications of lead titanate", Journal of Applied Physics 97 (3). 
[13]B. H. Rabee, M. A. Habeeb and A. Hashim ,2014 "Preparation of (PS-PMMA-ZnCl2) Composites and Study their Electrical and Optical Properties" , International Journal of Science and Research (IJSR) , (3) 10 .

[14] R. M. Ahmed and S.M. El-Bashir, 2011, " Structure and Physical Properties of Polymer Composite Films Doped with Fullerene Nanoparticles", International Journal of Photoenergy, Article ID 801409, 6.

[15] M. Rashidian and D. Dorranian, 2014, " Low-intensity UV effects on optical constants of PMMA film", Journal of Theoretical Applied Physics, 8-121.

[16] A. Benchaabane, Z. Ben Hamed, F. Kouki, M. A. Sanhoury, K. Zellama, A. Zeinert, and H. Bouchriha, 2014. "Performances of effective medium model in interpreting optical properties of polyvinylcarbazole:ZnSe nanocomposites", Journal of Applied Physics, 115.

[17] P. Chandra, P. Naveen, U. Sasikala, V.V.R.N. RAO and A.K. Sharma, 2012. "Investigations on lithium ion complexed polyvinyl chloride (PVC) solid polymer electrolyte films", Engineering Science and Technology:An International Journal, 2-5.

[18] S. Kramadhati, K. Thyagarajan, 2013. "Optical Properties of Pure and Doped (Kno3 \& Mgcl2) Polyvinyl Alcohol Polymer Thin Films", International Journal of Engineering Research and Development, 6(8): 15-18.

[19] S., A. Mohamed, A. Al-Ghamdi, G. Sharma, M. El Mansy, 2014. Effect of ethylene carbonate as a plasticizer on CuI /PVA nanocomposite: Structure, optical and electrical properties", Journal of Advance Research, 5: 79-86.

[20]A. Kurt, 2010. Influence of $\mathrm{AlCl} 3$ on the optical properties of new synthesized 3-armed poly(methyl methacrylate) films, Turk Journal Chem., 34: 67-79.

[21]H. Hoppe, N.S. Sariciftci and D. Meissner, 2002. "Optical Constants of Conjugated. Polymer/ Fullerene", Mol. Cryst. Liq. Cryst., 385: [233]/113-[239]/119.

[22] P.U. Asogwa, 2010 "Effect of deposition medium on the optical and solid state properties of annealed $\mathrm{MnO} 2$ thin films", Journal of Optoelectronics and Biomedical Materials, 2(3): 109-117.

[23] W. Al-Taay, M., Abdul Nabi, Rahimi M. Yusop, E. Yousif, Mudhaffar Abdullah, J. Salimon, N. Salih, and S. Irwan Zubairi, 2014. Effect of Nano ZnO on the Optical Properties of Poly(vinyl chloride) Films, International Journal of Polymer Science, Article ID 697809, 6. 